\title{
BTA Magnet Field Map Archive And MAD Model
}

\author{
J. W. Glenn
}

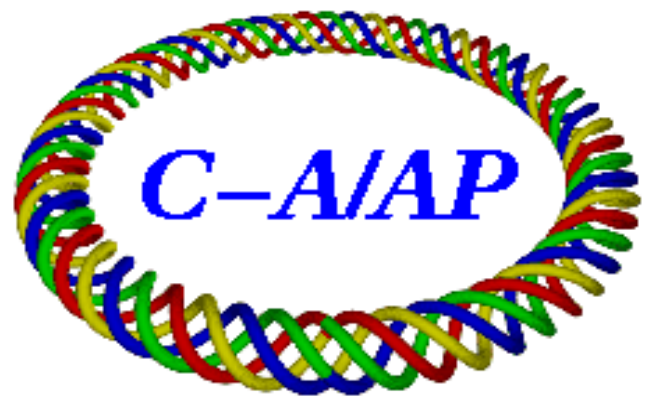

Collider-Accelerator Department Brookhaven National Laboratory

Upton, NY 11973

Notice: This document has been authorized by employees of Brookhaven Science Associates, LLC under Contract No. DEAC02-98CH10886 with the U.S. Department of Energy. The United States Government retains a non-exclusive, paid-up, irrevocable, world-wide license to publish or reproduce the published form of this document, or allow others to do so, for United States Government purposes. 


\section{BTA Magnet Field Map Archive \\ And MAD Model \\ J W Glenn \\ April 7, 2008}

This note publishes some and information that has resided in private files.

The attached tables were provided by Joseph Skelly from his archives. They show magnetic field measurements versus excitation current for the Booster to AGS transfer line quadropoles and dipoles based on field measurements [we believe] were done by the Magnet Division. Also given are Ed Blesser's fifth order fits of field versus current The results are given in 'Tesla' or T-M/M. These tables are attached to provide an archive of this data.

The MAD model of the BTA line does have the same values as shown in the attached fits so the transfer was correct. MAD uses as its 'gradient' for quads Tesla per meter normalized to rigidity [B-rho]. The model of the BTA line in use uses the T-M/M given in the tables divided by the length to give T/M which is then normalized by B-rho. Thus, the input to the model appears to be correct. The original model is also attached as part of a memo by Skelly describing it. 
TO: E. Auerbach, M. Blaskiewicz, E. Bleser, J.W. Glenn,

EROM: J. Skelly

TOPIC: BTA MAD Model in DSEE

DATE : $\quad$ 21-Apri1-1992

A copy of the MAD model for the Booster-To-AGS transfer line (BTA) as developed by Auerbach has been placed in a DSEE library. It is proposed that this library be regarded as the formal repository of the best current model. In their private copy(s); the lis the model, and may make any changes they please to competent in using implement modifications that such changes should be undertaken only to experimentation experimentation should be pursued in private copies.

\section{FEATURES OF INITIAL BTA MODEL}

The mad model lodged in the DSEE library is that developed by Auerbach after a critical study of the BTA line geometry, and includes quadrupole magnet excitation functions provided by Bleser.

- This model starts at a canonical location of the Booster F6 septurn, and does not address the question of a detailed model of the extraction trajectory within the septum; this issue is left for future improvements.

- - This model terminates at the small hole before the AGS A5 septum; realistic modeling beyond this point requires transport matrices that are currently under development. Moreover, the version of MAD currently in use does not left for future improvements.

O - This model declares the $S$ coordinate for all points of interest in the line, and derives drift lengths from these $S$ coordinates. The $S$ coordinates are specified in units of meters. The $S$ coordinates are derived by Auerbach from a critical study of BTA prints and other data, and are maintained in the booster model database. An explanation of their derivation will be the topic of a future technical note. - Drifts in this model are named according to the element upstream of the
drift.

O - Quadrupole strengths are parameterized in terms of the quadrupole currents, using a 4th-order power series expansion provided by Bleser; the parameters for the currents are initialized to zero.

O - The corrector kicks are set to zero; a future enhancement might parameterize these as a function of the corrector currents.

O - All markers have been denominated by their s coordinates measured in feet. - The subline organization terminates sublines at dipoles, except that a
subline termination is provided at the stripping foil. 
O - Three lines are defined for general modeling: BTA - F6 septum to small hole BTATOFL - F6 septum to stripping foil (BTA TO FoiL) BTAFMFL - stripping foil to small hole (BTA FroM FoiL)

O - No "USE" statement or other MAD action command (such as "TWISS", "MATCH" or "SURVEY") is specified in this model. Users are expected to append their own statements to implement such analyses as may suit them.

\section{USE OF DSEE}

The Domain Software Engineering Environment (DSEE) provides librarian type functions for maintenance of libraries of source files. Although intended for of source files, control system (AGSDCS) documentation and MAD models. The AGS Distributed files used to organize employs DSEE extensively to maintain many of the source to drive some of administer the control network and to provide data

Different libraries may be used by DSEE for different purposes. A library named "mad" has been provided for storage of MAD source codes and of MAD models. Files in this library may be read and copied by anyone, without even files to However, modification of these files, and addition of files to this library can be accomplished only by using DSEE. The "mad"

library may be found under the directory:

The BTA model within this/users/development/dsee/mad

The BTA model within this library is named: bta.model

DSEE permits elements in a library to be modified only by use of a reservation mechanism. When a user reserves an element from a library, that element is copied into the user's working directory, where the user may edit that copy ad the element is repla has finished whatever development was to be accomplished, one user, no other user may reserve the library. While an element is reserved by a "branch" on the element reserves an element, DSEE requirmit simultaneous development). When a user reservation. At any time therea input of a description of the reason for the element reserved and why (and when). Who may query DSEE to see who has an for reservation may be edited ton). When an element is replaced, the reason made. All replacements of development, including the user identityr kept as a permanent record of description. Each versiond change successive versions can be done to element is also preserved, and comparisons of version is identified both by a versiofy the changes that were made. Each

A user may run DSEE by entering into a shell the command:

\section{/com/dsee}

The first time you run DSEE you must specify: system, library, and model. Subsequent runs will recall this information from the last run. If you change library, and model for command need be entered to recover system, to be issued the first time you run when that system was used. The commands set system/users/develop mad system are: set system/users/development/dsee/mad_system set library /users/development/dsee/mad set model mad model

The above usage of the term model refers to what is called a DSEE model, and has nothing to do with a MAD model. A DSEE model describes to DSEE how each 
element in a library is to be processed. For program source codes, such processing would include appropriate compilation (using fortran, c or c+t
compilers for example).

The DSEE program provides extensive help facilities. Users wishing to develop the controls the use of DSEE should consult with their favorite programmer in 


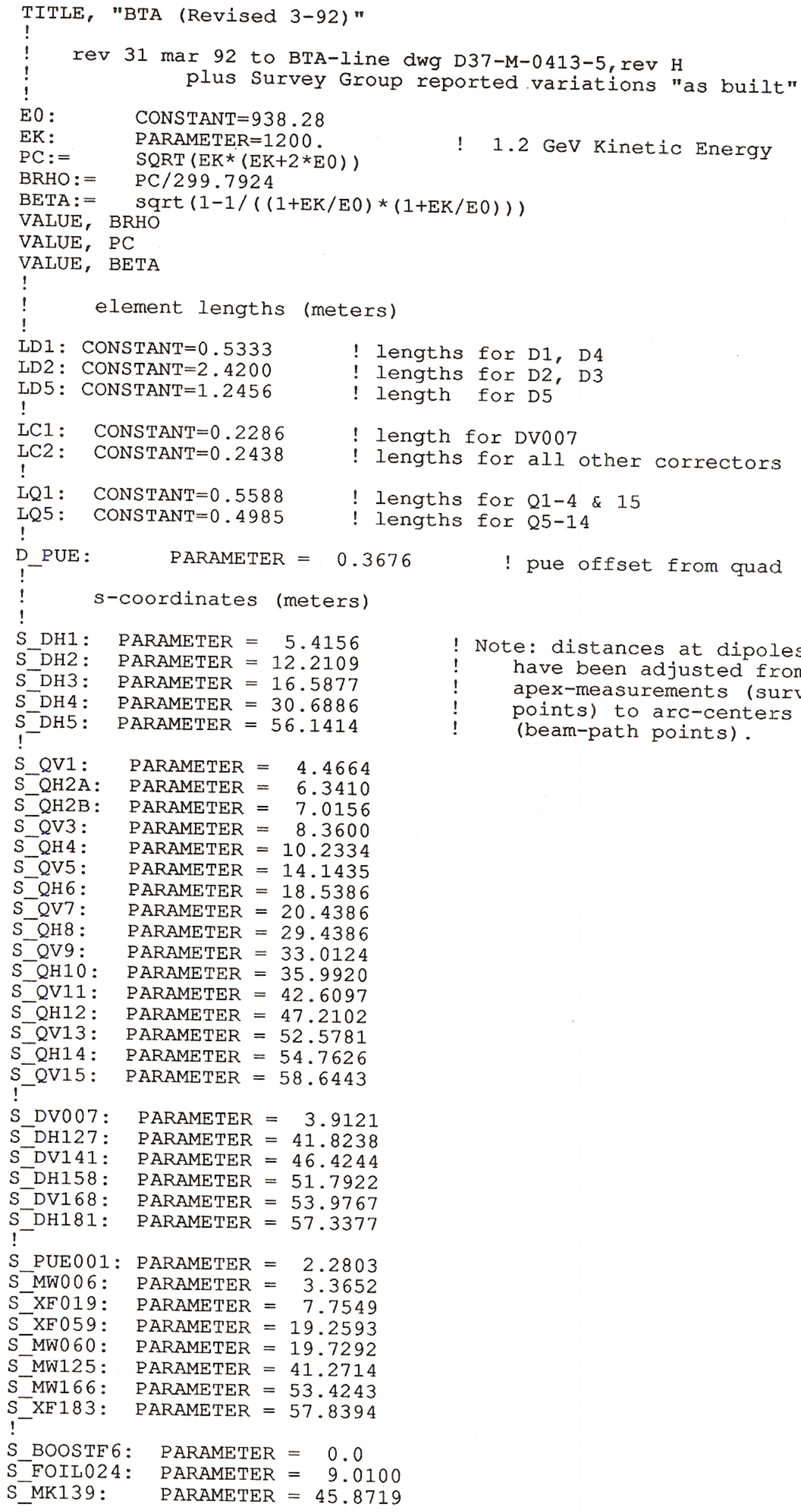




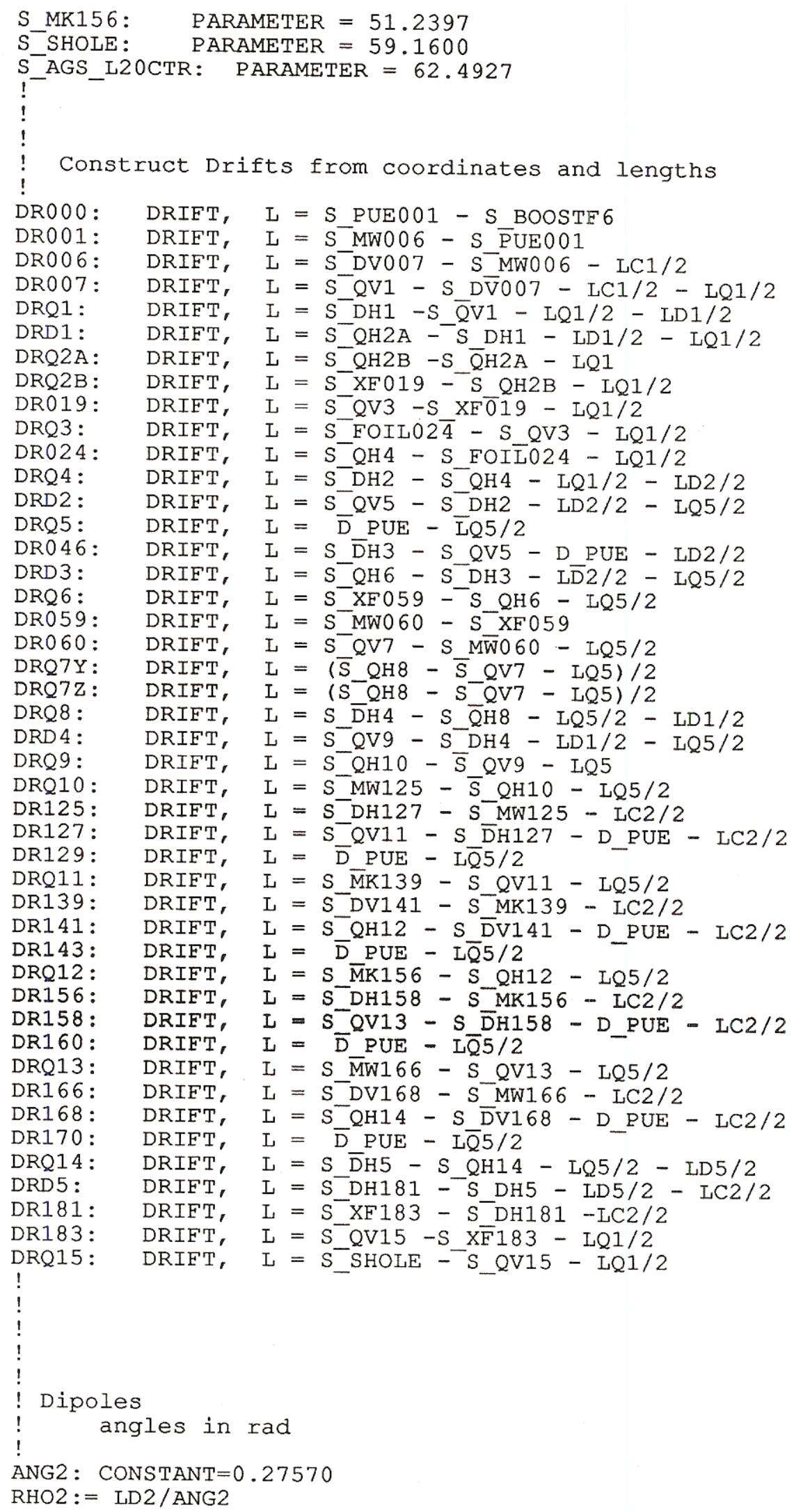
DH5: RBEND, L=LD5, ANGLE $=-0.14079$

!booster dipole
!booster dipole 
Quadrupoles

Strengths and Currents

$(\mathrm{T} / \mathrm{m}){ }^{*} \mathrm{~m}$ power series vs $I$, per Blesser $3 / 24 / 92$

$!$

$\mathrm{NQA}:=4.363 \mathrm{E}-02 \quad$ !Narrow, Q1-Q4, Q15

$\mathrm{NQC}:=6.309 \mathrm{E}-06$

$\mathrm{NQD}:=-8.609 \mathrm{E}-09$

$\mathrm{NQE}:=2.226 \mathrm{E}-12$

!

$\mathrm{BQA}:=1.907 \mathrm{E}-02$

$\mathrm{BQB}:=3.868 \mathrm{E}-03$

$\mathrm{BQC}:=2.538 \mathrm{E}-06$

$\mathrm{BQD}:=-2.952 \mathrm{E}-09$

$\mathrm{BQE}:=6.995 \mathrm{E}-13$

!

IQ1 $:=0.0$

IQ2 $:=0.0$

IQ3 $:=0.0$

IQ4 $:=0.0$

IQ5 $:=0.0$

IQ6 $:=0.0$

IQ7 $:=0.0$

IQ8 $:=0.0$

IQ9 $:=0.0$

$I Q 10:=0.0$

IQ11 $:=0.0$

IQ12 $:=0.0$

IQ13 $:=0.0$

IQ14 $:=0.0$

IQ15 $:=0.0$

!

! Quad Gradients

$Q 1 G:=(N Q A+I Q 1 *(N Q B+I Q 1 *(N Q C+I Q 1 *(N Q D+I Q 1 * N Q E)))) /[Q 1$

$Q 2 A G:=(N Q A+I Q 2 *(N Q B+I Q 2 *(N Q C+I Q 2 *(N Q D+I Q 2 * N Q E)))) / I Q 1$

$Q 2 B G:=(N Q A+I Q 2 *(N Q B+I Q 2 *(N Q C+I Q 2 *(N Q D+I Q 2 * N Q E)))) / L Q 1$

$Q 3 G:=(N Q A+I Q 3 *(N Q B+I Q 3 *(N Q C+I Q 3 *(N Q D+I Q 3 * N Q E)))) / I Q I$

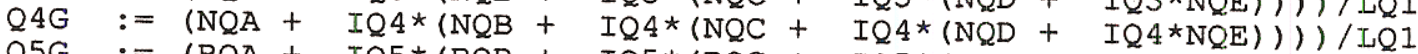

$Q 5 G:=(B Q A+I Q 5 *(B Q B+I Q 5 *(B Q C+I Q 5 *(B Q D+I Q 5 * B Q E)))) / L Q 5$

$Q 6 G:=(B Q A+I Q 6 *(B Q B+I Q 6 *(B Q C+I Q 6 *(B Q D+I Q 6 * B Q E)))) / L Q 5$

$Q 7 G:=(B Q A+I Q 7 *(B Q B+I Q 7 *(B Q C+I Q 7 *(B Q D+I Q 7 * B Q E)))) / L Q 5$

$\mathrm{Q} 8 \mathrm{G}:=(\mathrm{BQA}+\mathrm{IQ8*(BQB}+\mathrm{IQ8*(BQC}+\mathrm{IQ8*(BQD}+\mathrm{IQ8*BQE})))) / \mathrm{IQ} 5$

$Q 9 G:=(B Q A+I Q 9 *(B Q B+I Q 9 *(B Q C+I Q 9 *(B Q D+I Q 9 * B Q E)))) / I Q 5$

$Q 10 G:=(B Q A+I Q 10 *(B Q B+I Q 10 *(B Q C+I Q 10 *(B Q D+I Q 10 * B Q E)))) / I Q 5$

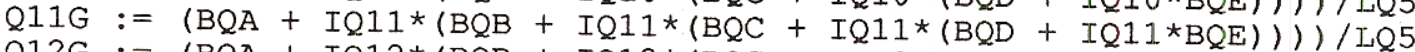

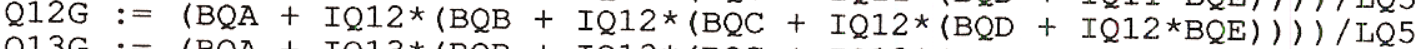

$\mathrm{Q} 13 \mathrm{G}:=(\mathrm{BQA}+\mathrm{IQ13}$ * $\mathrm{BQB}+\mathrm{IQ13}(\mathrm{BQC}+\mathrm{IQ13}(\mathrm{BQD}+\mathrm{IQ13}$ * $\mathrm{BQE})))) / \mathrm{LQ5}$

$\mathrm{Q} 14 \mathrm{G}:=(\mathrm{BQA}+\mathrm{IQ14}$ *(BQB $+\mathrm{IQ14} *(\mathrm{BQC}+\mathrm{IQ14}$ *(BQD + IQ14*BQE)) ) )/LQ5

$Q 15 G:=(\mathrm{NQA}+\mathrm{IQ15}$ *(NQB + IQ15*(NQC + IQ15*(NQD + IQ15*NQE)) ) $) / \mathrm{LQ1}$

QV1: QUAD, L=LQ1, $K 1=-Q 1 \mathrm{G} / \mathrm{BRHO}$

QH2A: QUAD, L=LQ1, $K 1=Q 2 A G / B R H O$

QH2B: QUAD, L=LQ1, $\mathrm{K} 1=\mathrm{Q} 2 \mathrm{BG} / \mathrm{BRHO}$

QV3: QUAD, L=LQ1, $\mathrm{KI}=-\mathrm{Q} 3 \mathrm{G} / \mathrm{BRHO}$

QH 4: QUAD, I $=\mathrm{LQ1}, \mathrm{K} 1=\mathrm{Q} 4 \mathrm{G} / \mathrm{BRHO}$

QV5: QUAD, L=LQ5, $\mathrm{K} 1=-\mathrm{Q} 5 \mathrm{G} / \mathrm{BRHO}$

QH 6: QUAD, L=LQ5, $\mathrm{K} 1=\mathrm{Q} 6 \mathrm{G} / \mathrm{BRHO}$

QV7: QUAD, L=LQ5, $\mathrm{K} 1=-\mathrm{Q} 7 \mathrm{G} / \mathrm{BRHO}$

QH8: QUAD, L=LQ5, $\mathrm{K} 1=\mathrm{Q} 8 \mathrm{G} / \mathrm{BRHO}$

QV9: QUAD, L=LQ5, $\mathrm{K} 1=-\mathrm{Q} 9 \mathrm{G} / \mathrm{BRHO}$

QH10: QUAD, L=LQ5, K1=Q10G/BRHO

QV11: QUAD, L=LQ5, $\mathrm{K} 1=-\mathrm{Q} 11 \mathrm{G} / \mathrm{BRHO}$

QH12: QUAD, L=LQ5, $\mathrm{K} 1=\mathrm{Q} 12 \mathrm{G} / \mathrm{BRHO}$

QV13: QUAD, L=LQ5, K1=-Q13G/BRHO

QH14: QUAD, L=LQ5, K1=Q14G/BRHO

QV15: QUAD, L=LQ1, K1=-Q15G/BRHO !

! Correctors 


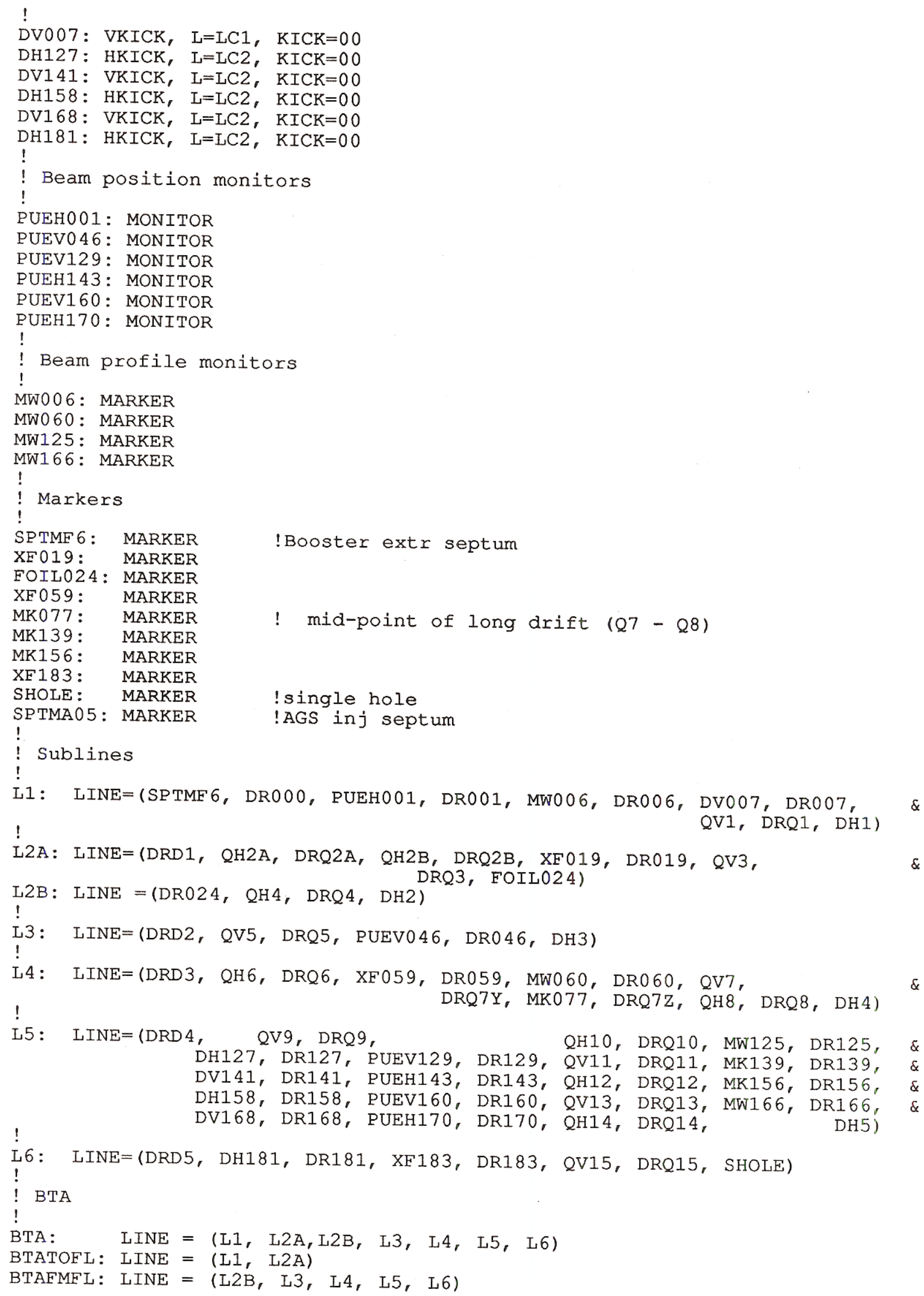


BTA MAGNETS

FIT TO FIELD SHAPE MEASUREMENTS

BTA NARROW QUAD

LOCATIONS: QH1,QH2A,QH2B,QV3,QH4,QV15

PREPARED: $3 / 24 / 92$, EJB
$5 / 2>/ 92$

new: $I \rightarrow B 1$ mep
RESULTS OF FITIING

$$
\begin{array}{lr} 
& \multicolumn{1}{r}{\text { COEFF }} \\
A= & 4.363 E-02 \\
B= & 9.266 \mathrm{E}-03 \\
\mathrm{C}= & 6.309 \mathrm{E}-06 \\
\mathrm{D}= & -8.609 \mathrm{E}-09 \\
\mathrm{E}= & 2.226 \mathrm{E}-12 \\
& \\
& \\
& \text { STDERROR } \\
\mathrm{A}= & 3.16 \mathrm{E}-02 \\
\mathrm{~B}= & 0.31 \mathrm{E}-03 \\
\mathrm{C}= & 1.09 \mathrm{E}-06 \\
\mathrm{D}= & 1.30 \mathrm{E}-09 \\
\mathrm{E}= & 0.50 \mathrm{E}-12
\end{array}
$$

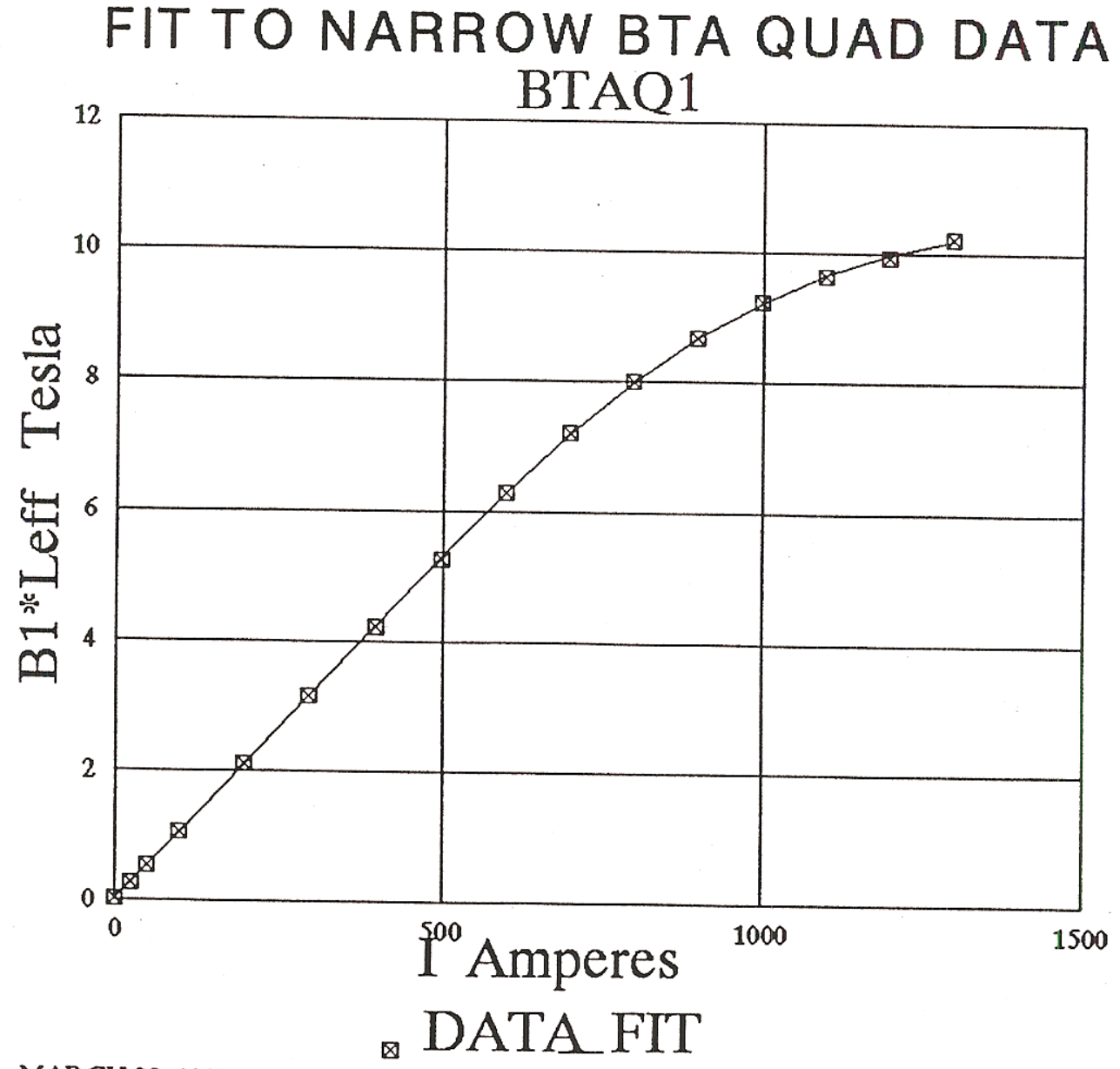




$$
\begin{aligned}
& \text { BTA MAGNETS } \\
& \text { FIT TO FIELD SHAPE MEASUREMENTS } \\
& \text { BTA NARROW QUAD } \\
& \text { LOCATIONS: QH1, QH2A, QH2B, QV3, QH4, QV15 } \\
& \text { PREPARED: } 5 / 18 / 92, \text { EJB } \\
& \text { LET } \mathrm{X}=\mathrm{B} 1 * \text { Leff } \\
& \mathrm{I}=\mathrm{A}+\mathrm{B} * \mathrm{X}+\mathrm{C} * \mathrm{X}^{\wedge} 2+\mathrm{D} * \mathrm{X}^{\wedge} 3+\mathrm{E} * \mathrm{X}^{\wedge} 4+\mathrm{F} * \mathrm{X}^{\wedge} 5
\end{aligned}
$$

DATA FOR BTAQ1

\begin{tabular}{rrr} 
B1*Leff & \multicolumn{1}{c}{ MEAS } & \multicolumn{1}{c}{ FIT } \\
Tesla & Amperes & \multicolumn{1}{c}{ A } \\
0.016 & 0.0 & -16.5 \\
0.265 & 24.6 & 16.3 \\
0.527 & 49.5 & 47.4 \\
1.053 & 99.4 & 102.7 \\
2.108 & 199.2 & 199.8 \\
3.160 & 298.7 & 295.7 \\
4.212 & 398.4 & 396.9 \\
5.257 & 498.2 & 500.4 \\
6.280 & 598.0 & 601.9 \\
7.213 & 697.5 & 697.9 \\
8.003 & 797.3 & 791.6 \\
8.675 & 897.1 & 892.6 \\
9.232 & 996.9 & 1002.0 \\
9.631 & 1096.5 & 1101.8 \\
9.943 & 1196.3 & 1195.9 \\
10.217 & 1296.1 & 1292.8
\end{tabular}

RESULTS OF FITTING

$$
\begin{array}{lr} 
& \multicolumn{1}{c}{\text { COEFF }} \\
A= & -18.752 \\
B= & 140.000 \\
C= & -31.868 \\
D= & 9.123 \\
E= & -1.150 \\
F= & 0.0539 \\
& \\
A= & \text { STD ERROR } \\
B= & 4.56 \\
C= & 11.93 \\
D= & 2.73 \\
E= & 0.27 \\
F= & 0.010
\end{array}
$$


BTA MAGNETS

FIT TO FIELD SHAPE MEASUREMENTS

BTA NORMAL QUAD

LOCATIONS: QV5,QH6,QV7,QH8,QV9,QH10,QV11,QH12,QV13,QH14

PREPARED: $3 / 24 / 92$, EJB

$B 1^{\star}$ Leff $=A+B * 1+C *$ ^ $2+D^{\star}\left|\wedge 3+E^{\star}\right| \wedge 4$

MEASURED DATAFOR

BTAQ05

\begin{tabular}{|c|c|}
\hline $\begin{array}{c}\text { I } \\
\text { Amperes }\end{array}$ & $\begin{array}{c}\text { B1 *Leff } \\
\text { Tesia }\end{array}$ \\
\hline 0.0 & 0.000 \\
\hline 24.7 & 0.112 \\
\hline 49.5 & 0.222 \\
\hline 99.2 & 0.443 \\
\hline 198.6 & 0.887 \\
\hline 397.3 & 1.774 \\
\hline 596.4 & 2.658 \\
\hline 795.1 & 3.511 \\
\hline 994.4 & 4.180 \\
\hline 1192.8 & 4.647 \\
\hline 1391.6 & 4.964 \\
\hline 1591.1 & 5.200 \\
\hline
\end{tabular}

RESULTS OF FITTING

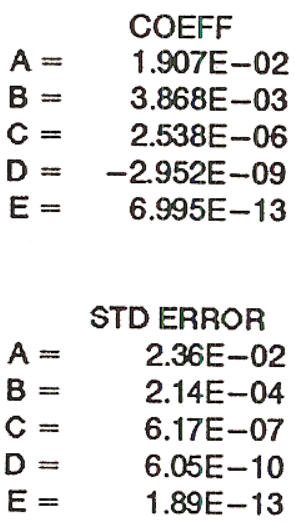

FIT TO NORMAL BTA QUAD DATA BTAQ05

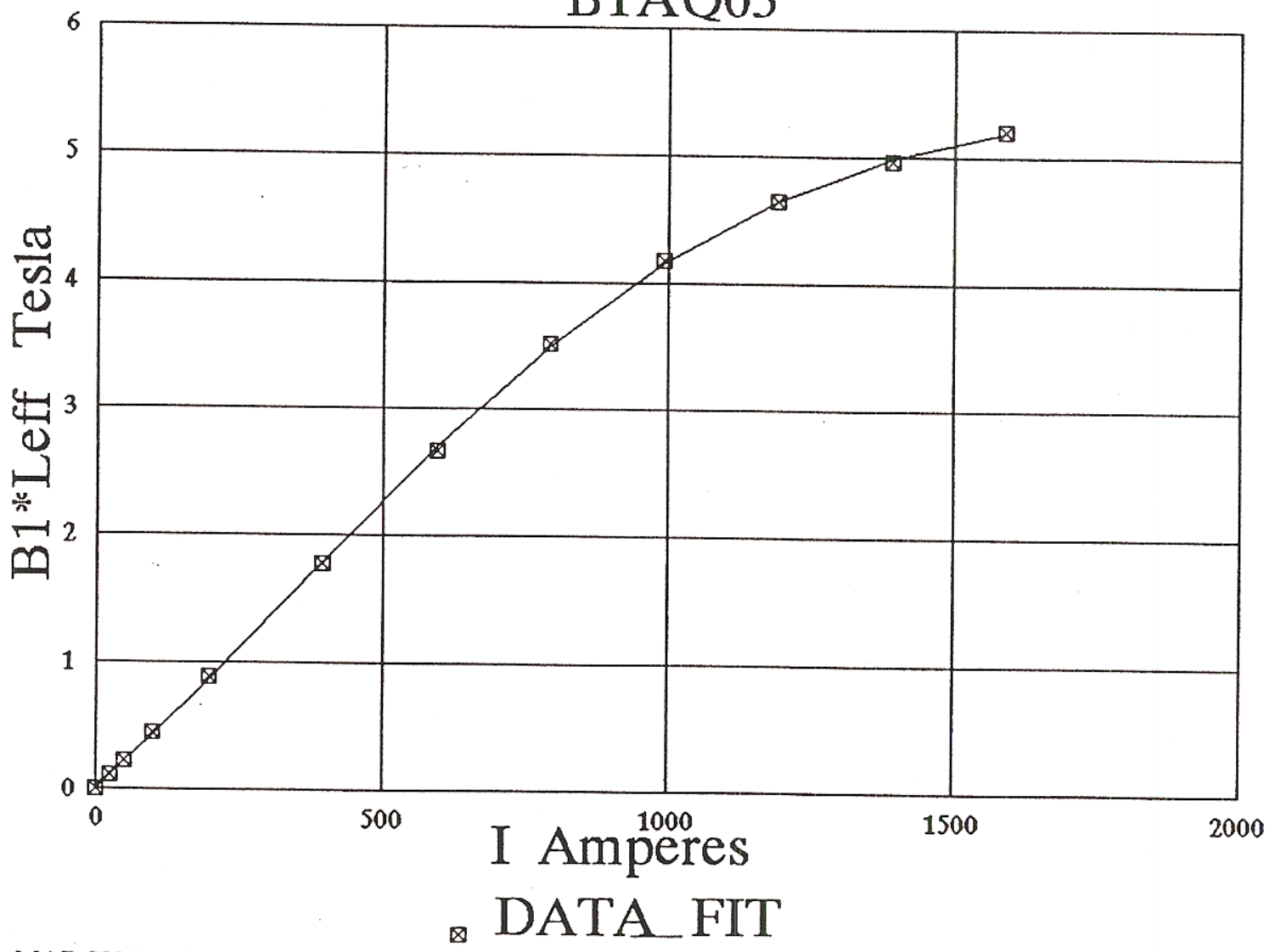




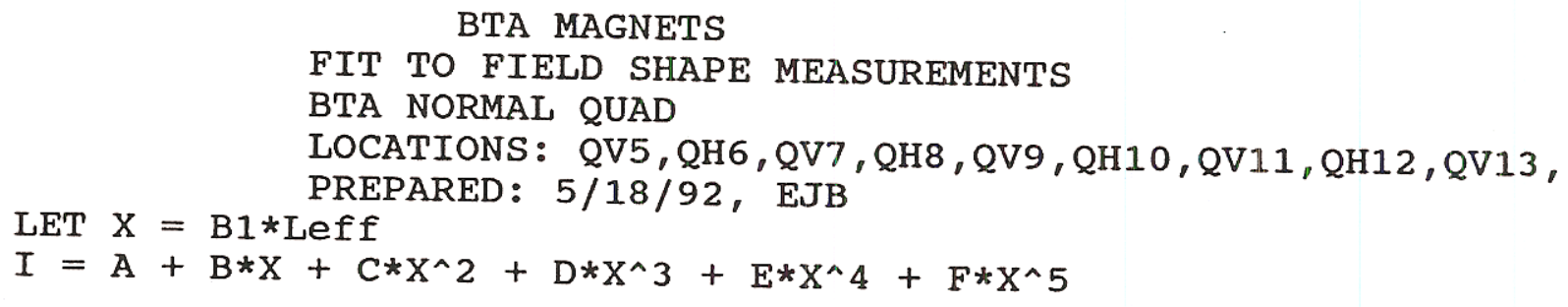

DATA FOR BTAQ05

\begin{tabular}{rrr} 
& \multicolumn{1}{c}{ MEAS } & \multicolumn{1}{c}{ FIT } \\
B1*Leff & \multicolumn{1}{c}{ I } & \multicolumn{1}{c}{ I } \\
Tesla & Amperes & \multicolumn{1}{c}{ Amperes } \\
0 & 0.0 & -8.967 \\
0.111513 & 24.7 & 20.719 \\
0.221845 & 49.5 & 48.484 \\
0.44326 & 99.2 & 100.744 \\
0.887082 & 198.6 & 198.733 \\
1.774034 & 397.3 & 395.580 \\
2.657603 & 596.4 & 598.009 \\
3.511294 & 795.1 & 796.234 \\
4.180247 & 994.4 & 990.584 \\
4.647032 & 1192.8 & 1194.419 \\
4.963837 & 1391.6 & 1393.833 \\
5.19978 & 1591.1 & 1589.472
\end{tabular}

RESULTS OF FITTING

$$
\begin{array}{lr} 
& \multicolumn{1}{c}{\text { COEFF }} \\
A= & -8.967 \\
B= & 274.726 \\
C= & -82.028 \\
D= & 52.747 \\
E= & -14.700 \\
F= & 1.504 \\
& \\
A= & \text { STD ERROR } \\
B= & 2.950 \\
C= & 29.534 \\
D= & 13.721 \\
E= & 2.777 \\
F= & 0.203
\end{array}
$$


BTA MAGNETS

FIT TO FIELD SHAPE MEASUREMENTS

BTA SHORT DIPOLE

LOCATIONSDHI, DH4

PREPARED: $3 / 24 / 92$, EJB

$B 0^{\star}$ Leff $=A+B^{\star}$ I $+C^{\star}\left|\wedge 2+D^{*}\right| \wedge 3+E^{\star} \mid \wedge 4$

MEASURED DATA FOR BTAD01

\begin{tabular}{rr}
\multicolumn{1}{l}{$\begin{array}{c}\text { Amperes } \\
\text { Amo*Leff }\end{array}$} & $\begin{array}{c}\text { Bo } \\
\text { Tesla*meters }\end{array}$ \\
0.0 & 0.0000 \\
24.4 & 0.0287 \\
49.3 & 0.0580 \\
99.2 & 0.1168 \\
199.0 & 0.2341 \\
298.6 & 0.3508 \\
398.2 & 0.4660 \\
498.0 & 0.5736 \\
597.8 & 0.6547 \\
697.3 & 0.7176 \\
797.0 & 0.7695
\end{tabular}

RESULTS OF FITTING

$$
\begin{array}{lr}
A= & \multicolumn{1}{c}{\text { COEFF }} \\
A= & 1.990 E-03 \\
B= & 1.071 E-03 \\
C= & 7.783 E-07 \\
D= & -1.531 E-09 \\
E= & 4.798 E-13 \\
& \\
& \\
A= & \text { STD ERROR } \\
B= & 3.28 E-03 \\
C= & 6.24 E-05 \\
D= & 3.51 E-07 \\
E= & 6.80 E-10 \\
& 4.23 E-13
\end{array}
$$

FIT TO 18 INCH BTA DIPOLE DATA BTAD01

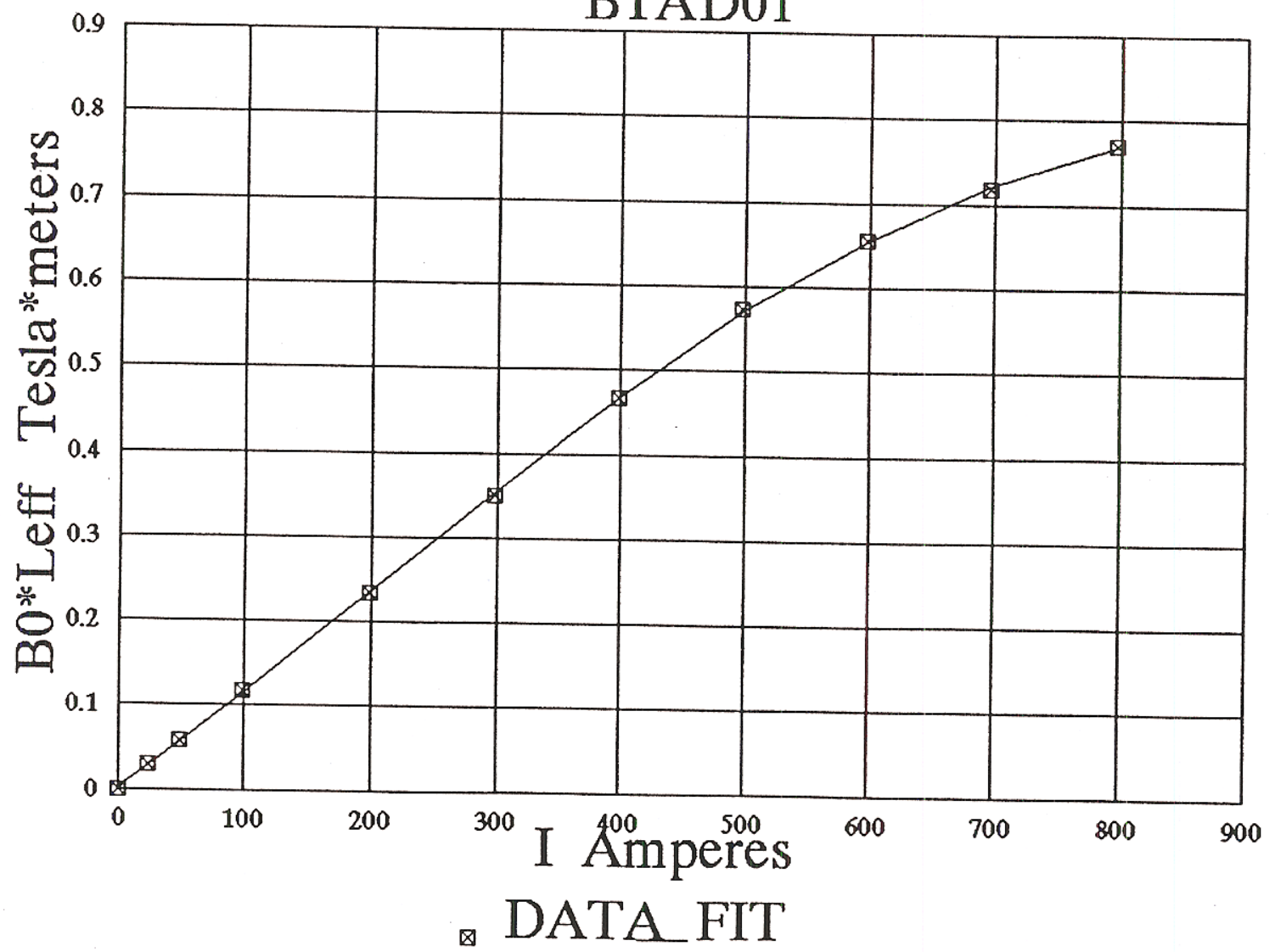




\section{BTA MAGNETS}

FIT TO FIELD SHAPE MEASUREMENTS

BTA SHORT DIPOLE

LOCATIDH1, DH4

PREPARED: 5/18/92, EJB

LET $X=B 0 *$ Leff

$I=A+B * X+C * X^{\wedge} 2+D * X \wedge 3+E * X^{\wedge} 4$

DATA FOR BTADO1

BO*Leff I

Tesla*met Amperes

$$
0
$$

0.028693

0.057981

0.11675

0.234087

0.350777

0.466035

0.57355

0.654678

0.71762

0.769532
0.00

24.37

49.32

99.21

199.02

298.61

398.15

497.96

597.77

697.26

797.03

\section{FIT}

I

Amperes

7.50

27.58

49.83

98.10

199.86

299.07

396.41

499.31

598.04

696.31

797.40
RESULTS OF FITTING
COEFF
$A=7.50$
$\mathrm{B}=664.69$
$C=1320.39$
$\mathrm{D}=-3504.62$
$E=3118.41$
STD ERROR
$A=1.45$
$B=63.43$
$C=300.22$
$D=532.77$
$E=316.42$ 
BTA MAGNETS

FIT TO FIELD SHAPE MEASUREMENTS

BTA LONG DIPOLE

LOCATIONS: DH5

PREPARED: 5/18/92, EJB

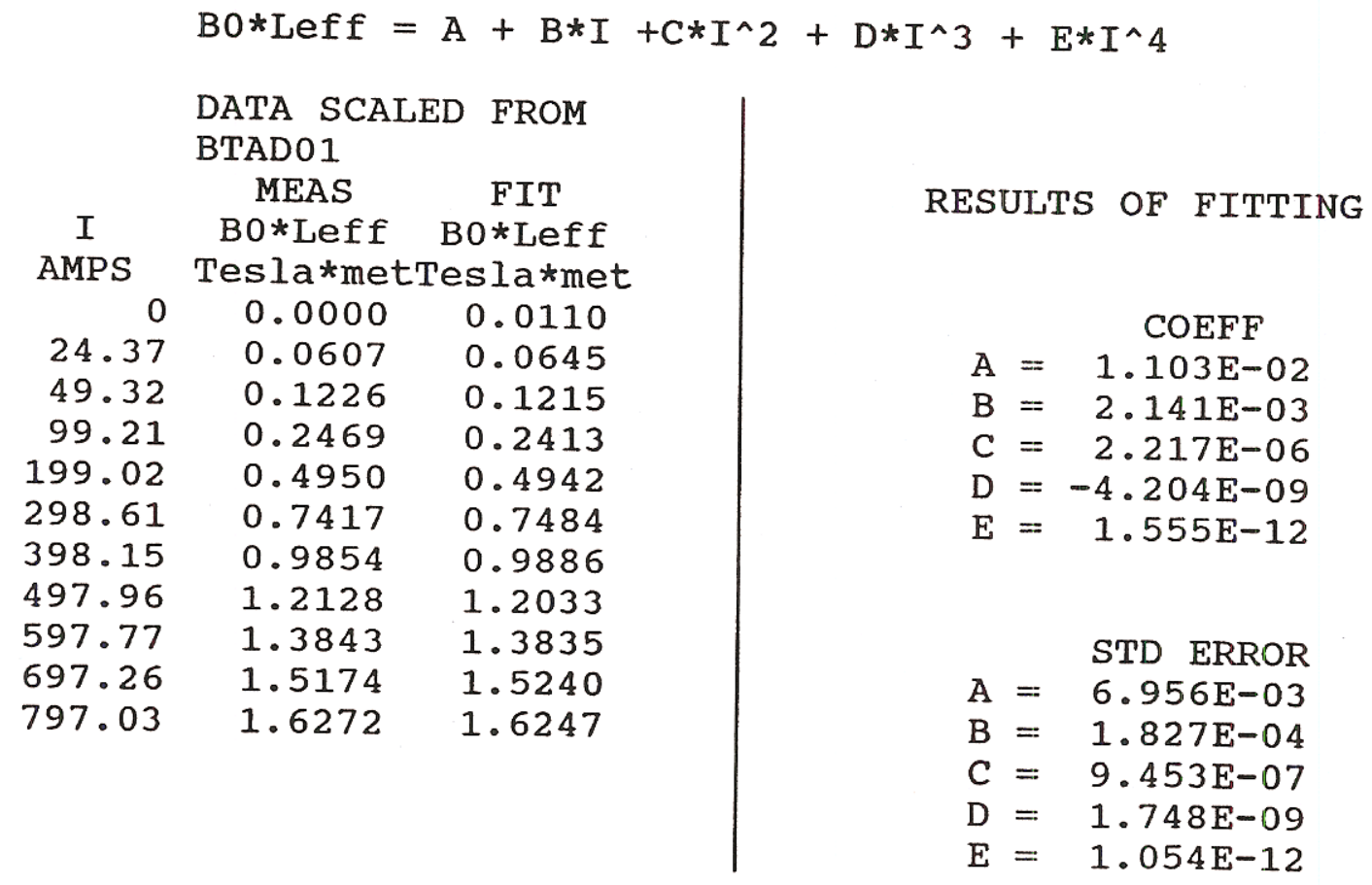


BTA MAGNETS

FIT TO FIELD SHAPE MEASUREMENTS BTA LONG DIPOLE LOCATIONS : DH5

PREPARED: 5/18/92, EJB

LET $X=B O *$ Leff

$I=A+B * X+C * X^{\wedge} 2+D * X \wedge 3+E * X \wedge 4$

DATA SCALED FROM

BTAD01

MEAS

$B 0 *$ Leff

Tesla*met Amperes

0.00

24.37

49.32

99.21

199.02

298.61

398.15

497.96

597.77

697.26

797.03

\section{FIT}

I

Amperes

3.81

25.27

48.58

98.16

200.39

299.04

396.08

499.22

598.25

696.51

797.21
RESULTS OF FITTING

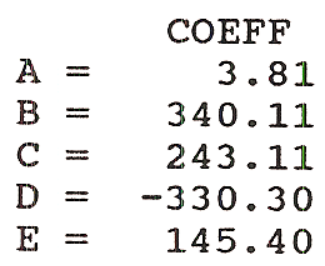

STD ERROR

$A=1.50$

$\mathrm{B}=21.19$

$C=52.13$

$\mathrm{D}=46.25$

$\mathrm{E}=13.48$ 
BTA MAGNETS

FIT TO FIELD SHAPE MEASUREMENTS

BTA SHORT CORRECTION MAGNET

LOCATIONSDVOO7

PREPARED: $3 / 24 / 92$, EJB

$B 0^{*}$ Leff $=A+B^{\star}\left|+C^{*}\right| \wedge 2$

MEASURED DATAFOR

BTAC01

\begin{tabular}{rr}
\multicolumn{1}{c}{$\begin{array}{r}\text { I } \\
\text { Amperes }\end{array}$} & $\begin{array}{c}\text { Bo'Leff } \\
\text { Tesla`meters }\end{array}$ \\
0.00 & $\mathrm{NA}$ \\
2.05 & 0.00154 \\
4.06 & 0.00281 \\
6.07 & 0.00413 \\
8.06 & 0.00544 \\
10.07 & 0.00678 \\
12.00 & 0.00808 \\
14.09 & 0.00945 \\
16.03 & 0.01071 \\
18.10 & 0.01205 \\
20.07 & 0.01332
\end{tabular}

RESULTS OF FITTING

$$
\begin{array}{lr}
A= & \multicolumn{1}{c}{\text { COEFF }} \\
A= & 1.586 E-04 \\
B= & 6.589 E-04 \\
C= & -9.611 E-08
\end{array}
$$

$\begin{array}{lr} & \text { STD ERROR } \\ A= & 2.48 E-05 \\ B= & 6.09 E-06 \\ C= & 2.68 E-07\end{array}$

\section{SHORT BTA CORRECTION MAGNET DAT} BTAC01

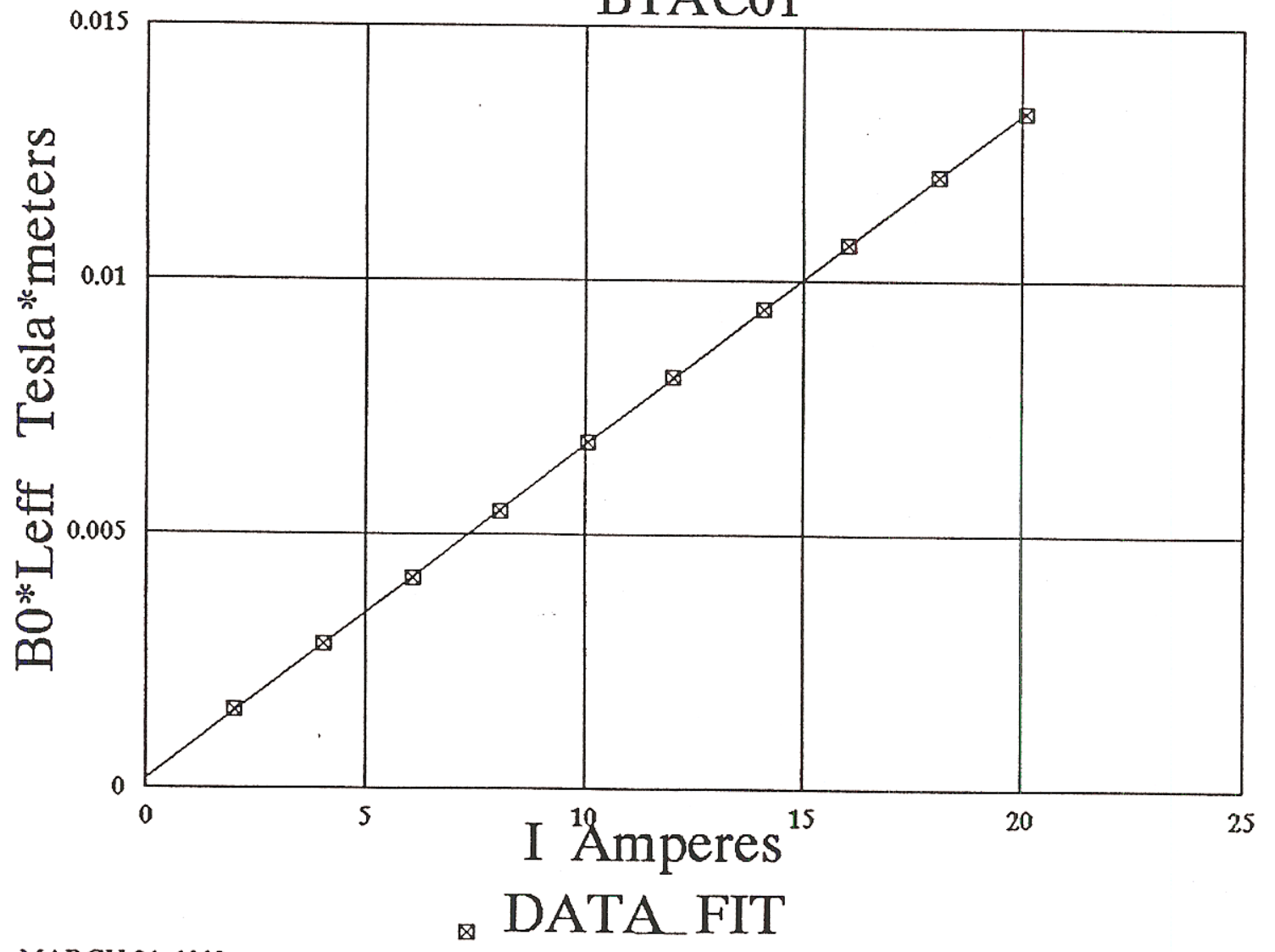


BTA MAGNETS

FIT TO FIELD SHAPE MEASUREMENTS

BTA SHORT CORRECTION MAGNET

LOCATIONS: DV007

$\mathrm{X}=\mathrm{B} 0 *$ Leff

PREPARED : 5/19/92, EJB

$I=A+B * X+C * X^{\wedge} 2$

MEASURED DATA FOR BTACO 1

BO*Leff

Tesla*met Amperes

NA

0.00154

0.002814

0.004128

0.005443

0.006777

0.008077

0.009446

0.010706

0.012052

0.013322
0.00

2.05

4.06

6.07

8.06

10.07

12.00

14.09

16.03

18.10

20.07
FIT

I

Amperes

2.0975

4.0332

6.0309

8.0294

10.0595

12.0401

14.1248

16.0465

18.0987

20.0385
RESULTS OF FITTING

$$
\begin{array}{lr} 
& \multicolumn{1}{c}{\text { COEFF }} \\
A= & -0.240 \\
B= & 1517.373 \\
C= & 354.740
\end{array}
$$
$A=S T D$ ERROR
$\mathrm{B}=14.555$
$C=956.178$ 
BTA MAGNETS

FIT TO FIELD SHAPE MEASUREMENTS

BTA NORMAL CORRECTION MAGNET

LOCATIONSDH1 27, DVI 41, DH158, DV168, DV181

PREPARED: $3 / 24 / 92$, EJB

$B 0^{*}$ Leff $=A+B^{\star}\left|+C^{\star}\right| \wedge 2$

MEASURED DATA FOR

BTACO2

\begin{tabular}{rr}
\multicolumn{1}{l}{$\begin{array}{c}\text { Bo`Leff } \\
\text { Amperes }\end{array}$} & $\begin{array}{r}\text { Bosla } \\
\text { Temeters }\end{array}$ \\
0.0 & NA \\
2.1 & 0.0018 \\
4.1 & 0.0033 \\
6.1 & 0.0048 \\
8.1 & 0.0063 \\
10.0 & 0.0078 \\
12.0 & 0.0093 \\
14.1 & 0.0109 \\
16.0 & 0.0122 \\
18.1 & 0.0137 \\
20.1 & 0.0150
\end{tabular}

RESULTS OF FITTING

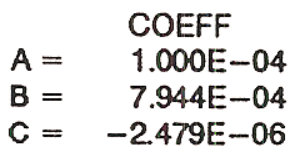

$$
\begin{array}{lr} 
& \text { STD ERROR } \\
\mathrm{A}= & 5.56 E-05 \\
\mathrm{~B}= & 1.37 \mathrm{E}-05 \\
\mathrm{C}= & 6.04 \mathrm{E}-07
\end{array}
$$

\section{NORMAL BTA CORRECTION MAGNET DA'} BTAC02

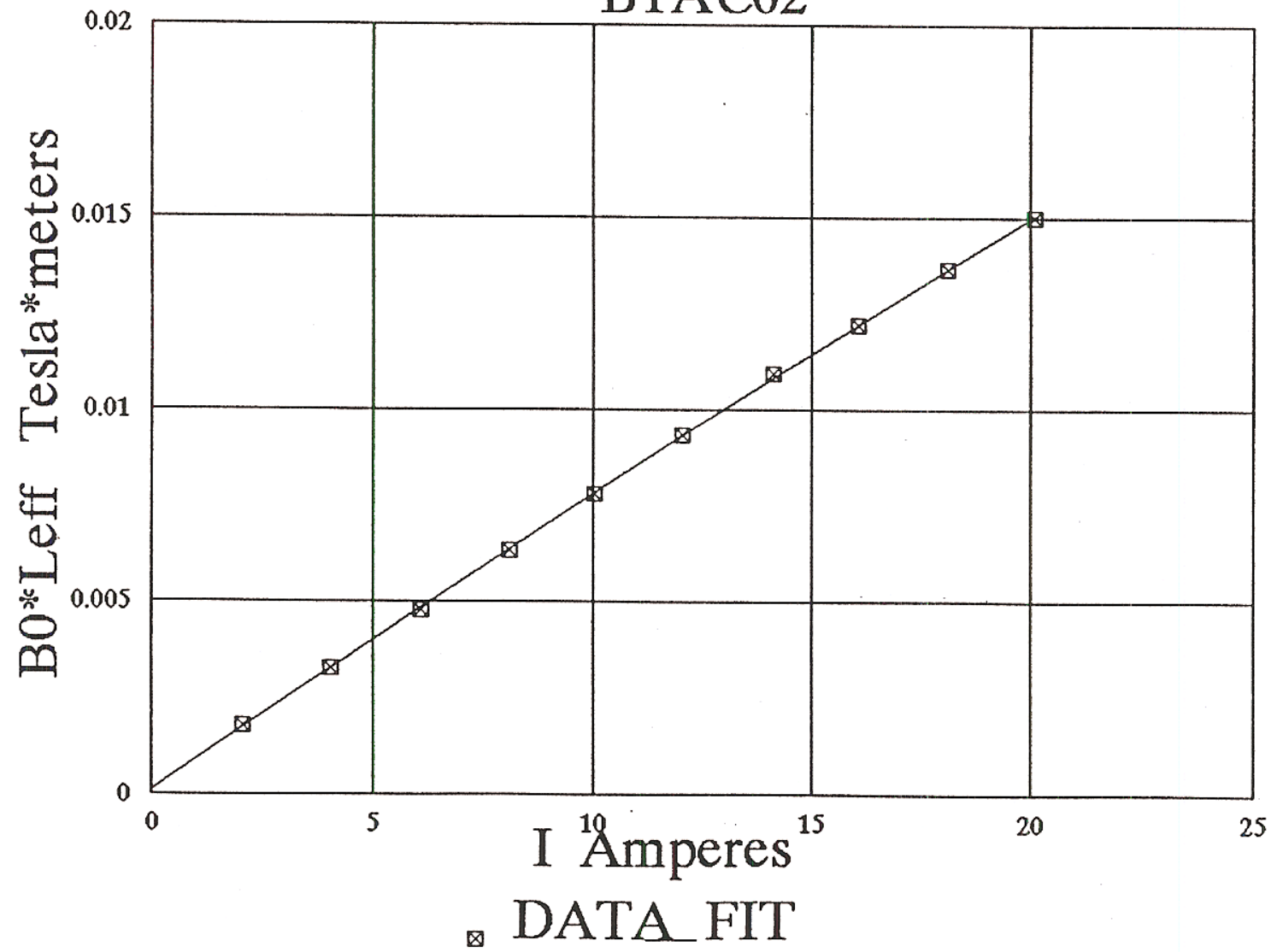




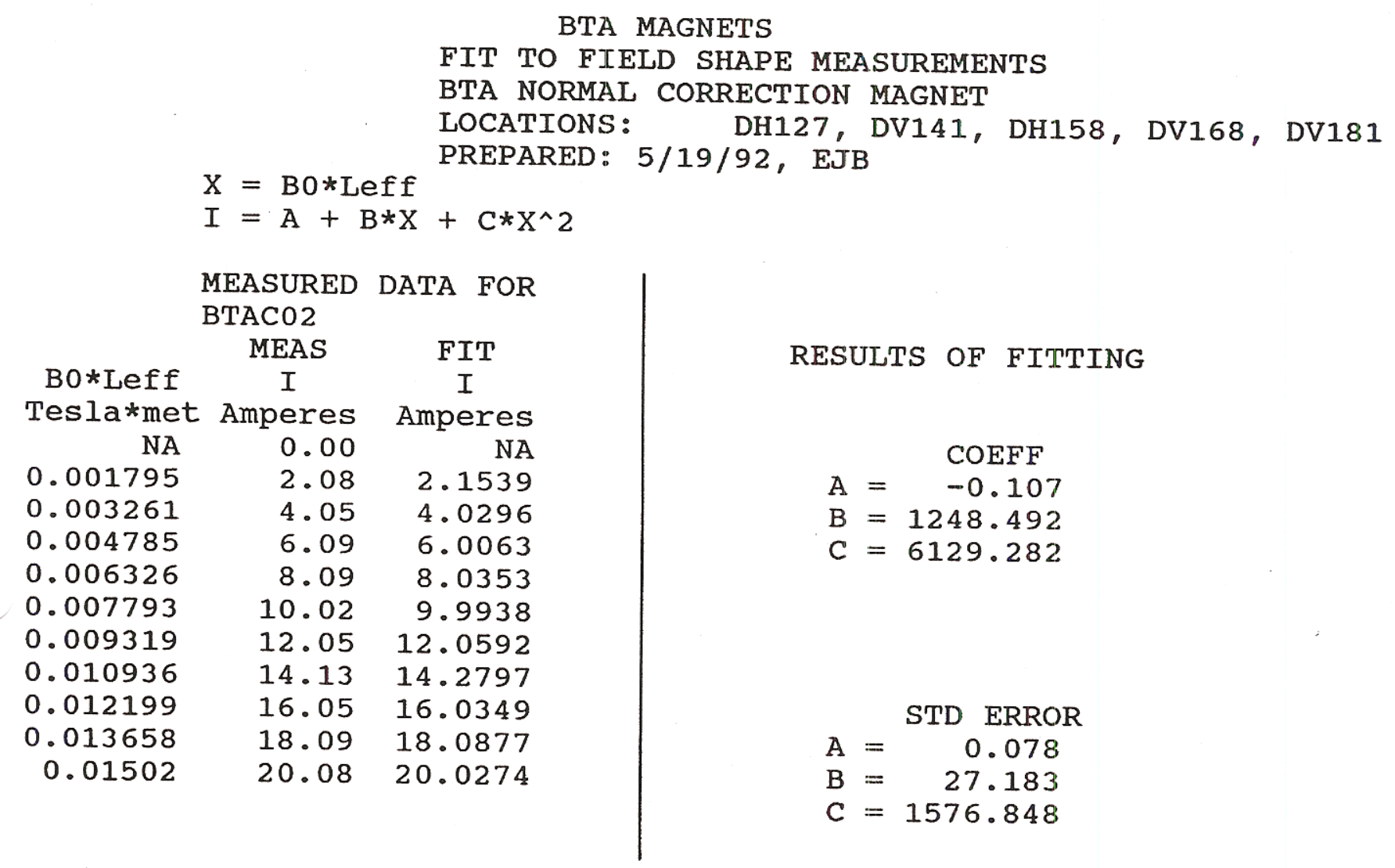




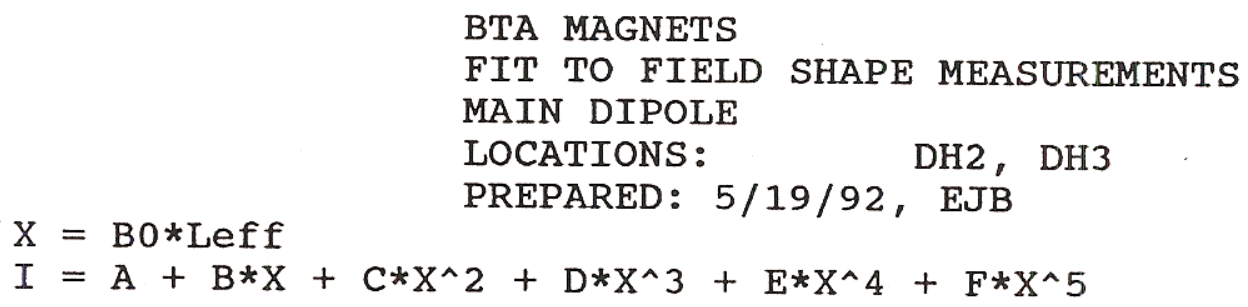

MEASURED DATA FOR BMDOOO

$\begin{array}{ccc} & \text { MEAS } & \text { FIT } \\ \text { BO*Leff } & \text { I } & \text { I } \\ \mathrm{T} * \mathrm{~m} & \text { AMPERES } & \text { AMPERES }\end{array}$

0.001791

0.030826

0.059972

0.118428

0.235754

0.353368

0.471099

0.588934

0.824388

1.059633

1. 294521

1.528859

1.762699

1.994625

2.223399

2.336137

2.446223

2.55121

2.650123

2. 740747

2.823939
$0-2.19451$

$50 \quad 49.14203$

$100 \quad 100.2885$

$200 \quad 201.8603$

$400 \quad 402.6912$

$600 \quad 601.5655$

800799.6678

$1000 \quad 997.9986$

14001396.611

$1800 \quad 1798.624$

$2200 \quad 2202.034$

$2600 \quad 2603.834$

30003002.666

$3400 \quad 3397.975$

$3800 \quad 3794.553$

$4000 \quad 3996.295$

$4200 \quad 4200.207$

$4400 \quad 4403.569$

$4600 \quad 4605.708$

$4800 \quad 4802.319$

$5000 \quad 4994.585$
RESULTS OF FITTING

COEFF

$$
\begin{aligned}
A= & -5.37434 \\
B= & 1776.103 \\
C= & -255.124 \\
D= & 301.8492 \\
E= & -146.187 \\
F= & 25.15767 \\
& \text { STD ERROR }
\end{aligned}
$$

$$
\begin{aligned}
& A=3.557049 \\
& B=23.25641 \\
& C=59.03624 \\
& D=56.31998 \\
& E=22.46429 \\
& F=3.172225
\end{aligned}
$$




\author{
BTA MAGNETS \\ FIT TO FIELD SHAPE MEASUREMENTS \\ MAIN DIPOLE \\ LOCATIONS: $\quad$ DH2, DH3 \\ PREPARED: 5/19/92, EJB
}

$B 0 * \operatorname{Leff}=A+B * I+C * I^{\wedge} 2+D * I^{\wedge} 3+E * I^{\wedge} 4+F * I^{\wedge} 5$

MEASURED DATA FOR BMDOOO

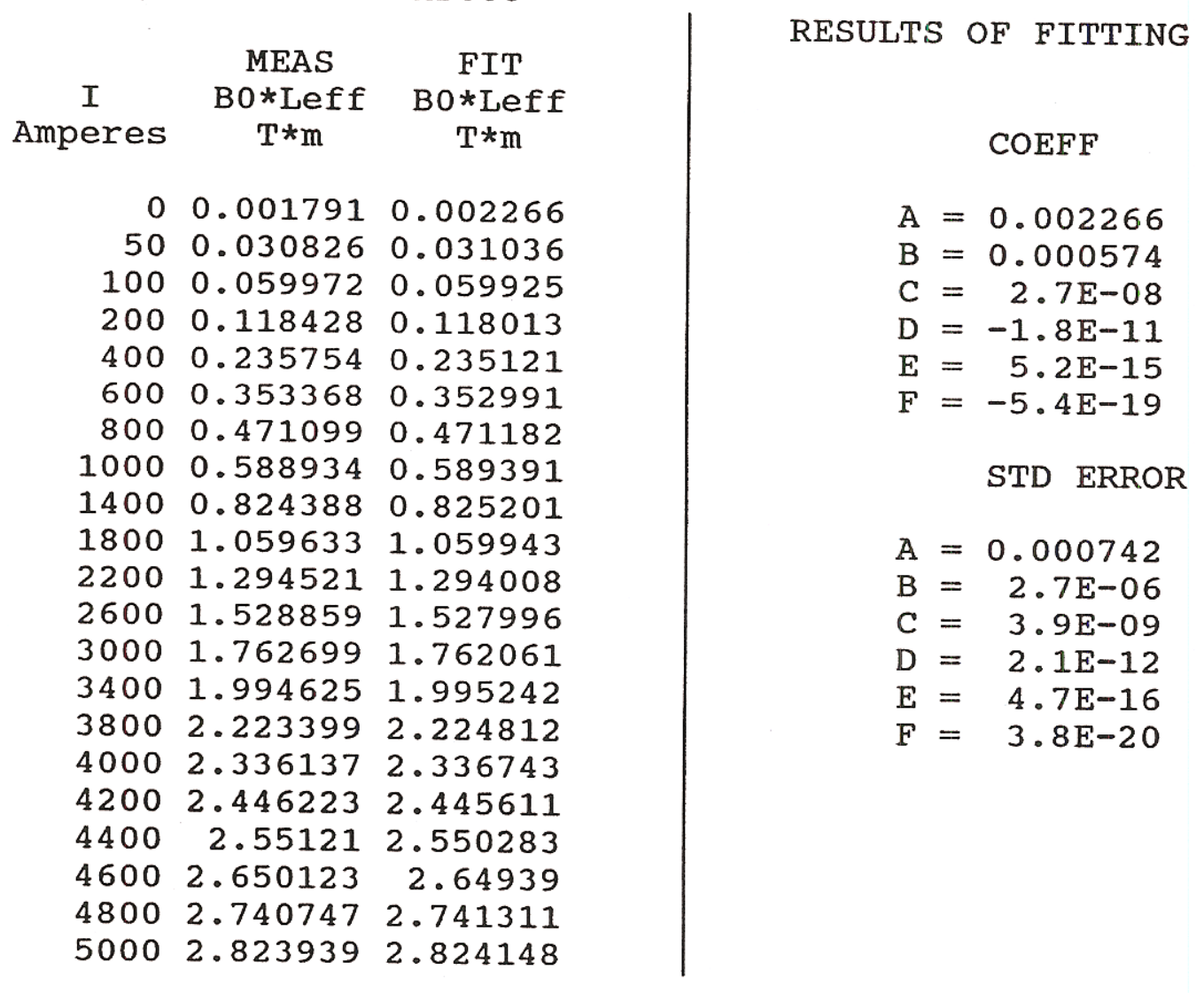




$\begin{array}{llll}\text { QH1 } & \text { ? } & & 9 " \\ \text { QH2A } & \text { BTAQ2A } & \text { N } & \\ \text { QH2B } & \text { BTAQ2B } & \text { N } & \\ \text { QV3 } & \text { BTAQV3 } & \text { N } & \\ \text { QH4 } & \text { BTAQV4 } & \text { N } & \\ \text { QV5 } & \text { BTAQ05 } & \text { B } & \\ \text { QH6 } & \text { BTAQ06 } & \text { B } & \\ \text { QV7 } & \text { BTAQ07 } & \text { B } & \\ \text { QH8 } & \text { BTAQ08 } & \text { B } & \\ \text { QV9 } & \text { BTAQ09 } & \text { B } & \\ \text { QH10 } & \text { BTAQ10 } & \text { B } & \\ \text { QV11 } & \text { BTAQ11 } & \text { B } & \\ \text { QH12 } & \text { BTAQ012 } & \text { B } & \\ \text { QV13 } & \text { BTAQ013 } & \text { B } & \\ \text { QH14 } & \text { BTAQ014 } & \text { B } & \\ \text { QV15 } & \text { BTAQ15 } & \text { N } & \\ & & & \\ \text { DH1 } & \text { BTAD01 } & & 18 " \\ \text { DH2 } & \text { BMD06 } & \text { B } & \\ \text { DH3 } & \text { BMD00 } & \text { B } & \\ \text { DH4 } & & & 18^{\prime \prime} \\ \text { DH5 } & \text { BTAD5 } & & 44^{\prime \prime} \\ & & & \\ & & & \\ \text { DV007 } & \text { ? } & & \\ \text { DH127 } & \text { BTAC06 } & & 9.5^{\prime \prime} \\ \text { DV141 } & \text { BTAC05 } & & 9.5^{\prime \prime} \\ \text { DH158 } & \text { BTAC07 } & & 9.5^{\prime \prime} \\ \text { DV168 } & \text { BTAC03 } & & 9.5^{\prime \prime} \\ \text { DV181 } & \text { BTAC02 } & & 9.5^{\prime \prime}\end{array}$

